\title{
« Etat » : définitions et réalités
}

\section{Bernard Vonglis}

\section{(Q) OpenEdition}

Journals

Édition électronique

URL : http://journals.openedition.org/plc/321

DOI : $10.4000 /$ plc.321

ISSN : 2117-5209

\section{Éditeur}

L'Harmattan

Édition imprimée

Date de publication : 1 janvier 2000

Pagination : 27-57

ISSN : 1279-8657

\section{Référence électronique}

Bernard Vonglis, « «Etat » : définitions et réalités », Pouvoirs dans la Caraibe [En ligne], 12 | 2000, mis en ligne le 14 mars 2011, consulté le 01 mai 2019. URL : http://journals.openedition.org/plc/321 ; DOI : $10.4000 /$ plc. 321 


\section{« Etat » : définitions et réalités}

par Bernard VONGLIS

"Toute définition juridique est hasardeuse, car le risque est grand qu'elle soit bouleversée »

JAVOLENUS, jurisconsulte romain

Parmi les notions d'usage quotidien dans les conversations, la presse écrite ou parlée, il n'en est pas de plus malaisée à définir avec précision que celle qu'exprime le mot «Etat». Et pourtant, à l'exception des étudiants en droit et en science politique qui doivent l'affronter dès le début de leur cursus, bien peu parmi ceux qui le prononcent ou l'entendent sont conscients de cette difficulté. Il existe de ce mot à peu près autant de définitions que de spécialistes qui en donnent, et si différentes qu'après en avoir pris connaissance l' « honnête homme » éprouve nécessairement un grand désarroi. On en jugera par trois exemples : pour G. Burdeau, l'Etat c'est « le pouvoir institutionnalisé »; selon $\mathrm{Cl}$. Leclercq, « une personne morale titulaire de la souveraineté »; quant aux internationalistes, ils y voient «une collectivité qui se compose d'un territoire et d'une population soumise à un pouvoir politique organisé $» 1$.

Ces définitions suggèrent deux réflexions.

1. G. Burdeau, Traité de science politique, $3^{\mathrm{e}}$ éd., Paris, 1969, t. 2, L'Etat, p. 169 ; Cl. Leclercq, Droit constitutionnel et institutions politiques, $9^{e}$ éd. Paris, 1995, p. 56; Avis $\mathrm{n}^{\circ} 1$ de la commission d'arbitrage, Conférence pour la paix en Yougoslavie, cité par J.-D. Mouton, «L'Etat selon le droit international», in L'Etat souverain à l'aube du XXI siècle, Ste FSE pour le droit international, Colloque de Nancy, Paris, 1994, p. 80. La plupart des données utilisées ci-après concernant l'Etat et la souveraineté pendant la 2 moitié du $\mathrm{XX}^{e}$ siècle jusqu'en 1994 proviennent de cet excellent ouvrage. 
On notera d'abord que chaque spécialiste privilégie l'aspect de la notion d'Etat qui correspond à son point de vue propre. Formulée par un politologue, la première est politique. La deuxième est à l'évidence l'œuvre d'un juriste. Mais toutes deux présentent un point commun, leur caractère abstrait. La troisième, au contraire, se veut concrète. Elle émane d'hommes soucieux de fixer avec précision la liste des éléments constitutifs d'un Etat existant ou à créer et d'organiser ses rapports avec les autres Etats.

On remarquera ensuite que le mot «Etat»y est pris dans deux sens différents quoique étroitement liés. La première se limite à ce que l'on appelle couramment « l'Etat»-pour le qualifier de monstre froid ou déclarer qu'il paiera - c'est-à-dire l'appareil de gouvernement ou d'administration, les «pouvoirs publics». Les deux autres mentionnent, outre celui-ci, un élément juridique - une personne morale - ou des éléments concrets - territoire, population - qui nous font connaître ce qu'est «UN Etat ». UN Etat est gouverné par l'Etat. Aucun des deux ne peut exister sans l'autre, mais il importe d'être bien conscient de celui auquel on se réfère quand on traite de cette notion.

A l'évidence donc, les définitions de l'Etat qui négligent le territoire et la population pèchent par insuffisance. Contrairement à l'opinion de Kelsen pour qui l'Etat n'est qu'un ensemble de normes juridiques ${ }^{2}$, il s'agit bien d'éléments constitutifs et non de conditions d'existence de celui-ci. Soyons clair : territoire et population sont les conditions d'existence de la souveraineté qu'exerce l'Etat et qui l'habilite à édicter des normes juridiques; territoire, population, et souveraineté sont les trois éléments constitutifs d'UN Etat. Kelsen réduit abusivement sa définition du mot «Etat » à celle de l'Etat. En droit privé, une personne morale est un groupement de personnes et de biens ${ }^{3}$. Pourquoi en serait-il autrement en droit public ? «Personne n'a jamais vu l'Etat» écrit G. Burdeau ${ }^{4}$. Bien au contraire, chaque

2. H. Kelsen, « Aperçu d'une théorie générale de l'Etat », RDP, 1926, p. 561 et ss.

3. G. Wicker, Personne morale, Rép. civ. Dalloz, Paris, 1998, p. 2.

4. G. Burdeau, L'Etat, Paris, 1970, p. 7. Pour le même auteur, Traité..., op. cit., t. 2 , L'Etat, p. 157, en totalisant « un territoire, une nation et une puissance de domination» on aboutit «à une notion bâtarde ». Et de donner un exemple : « En additionnant des 
Français le voit quotidiennement : il vit sur un territoire occupé depuis des millénaires et maintenant bien délimité, où il est entouré en majorité de Français; il voit à la télévision le président de la République, le gouvernement, le parlement... ; dans la rue ou dans son bureau, le gendarme, le douanier, le percepteur...

Aussi ne peut-on qu'approuver le souci de $\mathrm{Ph}$. Sueur de donner du mot «Etat» une définition complète et détaillée en réalisant la synthèse des trois précédentes: «Un territoire, un peuple, un gouvernement institutionnalisé et indépendant, réunis pour former cet être juridique doté de la puissance souveraine qui structure et contient la société politique $\gg^{5}$.

Cette définition conciliante pose toutefois un problème. En incluant celle de G. Burdeau, elle réduit à tort le domaine d'application de la notion d'Etat. Par «pouvoir institutionnalisé » cet auteur entendait en effet un pouvoir non seulement codifié par une constitution mais aussi détaché de la personne de celui ou de ceux qui l'exercent.

Admettant cette exigence fondamentale, d'autres auteurs vont plus loin en exigeant que ce pouvoir dépersonnalisé respecte les principes démocratiques, le droit, et singulièrement les droits de l'homme. Pour eux, la notion d'Etat se réduit donc à celle d'Etat de droit.

Ces restrictions paraissent devoir être écartées. La France des $\mathrm{XVII}^{\mathrm{e}}$ et $\mathrm{XVIII}{ }^{\mathrm{e}}$ siècles, époque où le mot abonde dans la législation comme dans les écrits des théoriciens, n'aurait pas été un Etat ? Et la France napoléonienne? Ou, plus près de nous, l'Allemagne

moutons avec des moutons, on obtiendra un troupeau, mais en ajoutant le pré et le berger le résultat ne sera qu'une notion confuse et inutilisable ». Bien au contraire, car, comme dans la définition de l'Etat, il ne s'agit pas d'additionner mais de combiner des éléments à l'évidence disparates mais complémentaires. Cette combinaison crée une entreprise agricole spécialisée dans l'élevage, un groupement destiné à réaliser des profits.

5. Ph. Sueur, Histoire du droit public français, $\mathrm{XV}^{\mathrm{e}}-\mathrm{XVII}^{\mathrm{e}}$ siècle, «La genèse de l'Etat contemporain », t. 1, La constitution monarchique, Paris, 1989, p. 71. 
hitlérienne ${ }^{6}$, l'URSS... ? Ou encore, en l'an 2000, les nombreux pays membres de l'ONU quoique gouvernés plus ou moins tyranniquement? En réalité, l'Etat de droit parfait n'existe pas et n'existera jamais parce que la notion et singulièrement sa composante principale, les droits de l'homme, évolue sans cesse, et que l'Etat de droit du moment a toujours un temps de retard sur elle. En outre, il n'y a pas de coupure nette entre Etat de droit et Etat qui ne mérite pas ce label, mais une gradation continue de la démocratie idéale à la tyrannie la plus brutale. Lorsque J. Bodin qualifie la République de «droit gouvernement », il précise que cette exigence n'exclut que les troupes de brigands ${ }^{7}$. Pour lui, l'Empire perse était un Etat. Cette conception serait-elle dépassée ? Il ne semble pas puisque la Conférence pour la sécurité et la coopération en Europe déclarait en 1990 que «la démocratie est un élément inhérent à l'Etat de droit »" à l'Etat de droit, et non pas à l'Etat en général ${ }^{9}$.

6. Voir M. Troper, «Y a-t-il un Etat nazi? », in Pour une théorie juridique de l'Etat, Paris, 1994, p. 177 et ss, qui après avoir fait une analyse à juste titre sévère des conditions de fonctionnement de l'Allemagne à cette époque, semble, si nous l'avons bien compris, finir par admettre que l'Etat nazi, à défaut d'être, à l'évidence, un Etat de droit, constituait « une espèce particulière du genre Etat », même si le titre du livre de M. Broszat, L'Etat hitlérien : L'origine et les structures du Troisième Reich, Paris, 1985, dont cet article est le compte-rendu, n'implique pas nécessairement cette idée. Les puissances européennes traitaient avec le Reich d'Etat à Etat, même si la suite a prouvé qu'elles avaient eu tort de le faire. Toute tentative pour rejeter la théorie positiviste de Duguit est vouée à l'échec. G. Burdeau ne donne pas la définition de l'Etat en général, mais celle de l'Etat... où le pouvoir est institutionnalisé. Celui-ci englobe l'Etat de droit et une partie de ceux ou les droits de l'homme ne sont pas respectés, l'autre partie étant constituée par les Etats tyranniques, tels le $\mathrm{III}^{\mathrm{e}}$ Reich, l'URSS, la Chine, etc...

7. J. Bodin, Les six livres de la République, $10^{\mathrm{e}}$ ed., 1593, L $1^{\mathrm{er}}$, ch 1 . On constatera par la suite que la définition des internationalistes est tout simplement celle de Bodin.

8. Cité par H. Thierry, «L'Etat et l'organisation de la société internationale », in L'Etat souverain..., op. cit., p. 198.

9. L'Europe est à cet égard un club plus fermé que l'ONU. Elle n'admet en son sein que des Etats de droit, comme vient de le confirmer l'attitude de certains membres à l'égard de l'Autriche. Parce qu'insuffisamment démocratique, la Turquie est toujours en attente. 
Quant à l'institutionnalisation du pouvoir, ce n'est qu'une illusion. L'histoire le prouve, la souveraineté est nécessairement personnifiée par le plus petit nombre possible de personnes : celles qui détiennent effectivement le pouvoir ${ }^{10}$. Le roi sous l'Ancien Régime, la Législative de 1791 à 1792 , le Comité de salut public en 1793 et 1794... Comme l'avoua Sieyès après cette expérience malheureuse dont il portait en grande partie la responsabilité, la souveraineté est une notion «monacale ». Peut-on accuser G. Burdeau de faire preuve de cynisme lorsqu'il écrit : «L'Etat est l'artifice qui permet de voir dans la puissance publique autre chose que la volonté propre des gouvernants... le fondement neutre de la puissance de domination $»^{11}$ ? Non, l'éminent politologue ne fait que constater une réalité que chaque citoyen peut vérifier quotidiennement.

Institutionnalisation du pouvoir, République et démocratie conviennent à la notion d'Etat, il est même hautement souhaitable qu'elles soient réunies. Mais elles ne sont pas indispensables à son existence. En d'autres termes, elles sont de la nature de l'Etat, pas de son essence.

Compte tenu de ces remarques, il convient, semble-t-il, de retenir une définition du mot «Etat » qui soit aussi compréhensive que possible, sans cesser d'être rigoureuse: Une personne morale regroupant une population et un territoire stables et dotée de la souveraineté.

Il paraît superflu d'insister sur la notion purement juridique de personne morale. Un groupement de personnes et de biens - dont le territoire - doté de la souveraineté est une personne morale qualifiée d'« Etat ». Dans le cas contraire, il ne mérite pas cette qualification. Il n'y a rien de plus à dire compte tenu du sujet traité. En revanche, territoire et surtout population et souveraineté peuvent poser problème. L'objet de cet exposé n'est pas de reprendre le sempiternel refrain sur

10. Sur cette question, voir B. Vonglis, «Souveraineté de la nation et souveraineté du peuple », in Nonagesimo anno, Mélanges en hommage à J. Gaudemet, Paris, 1999, p. 710-712 et la bibliographie citée.

11. G. Burdeau, Traité..., op. cit., t. 2, p. 47, n. 98. 
ces trois éléments ${ }^{12}$ mais de montrer, après l'avoir brièvement fredonné, qu'il s'agit d'un leitmotiv pas toujours respecté. Que la réalité ne confirme pas toujours la théorie, qu'elle la respecte même de moins en moins.

On examinera donc d'abord les éléments qui constituent le groupement «Etat» (I), puis l'autorité que ce groupement exerce sur lui-même et qui le rend indépendant, la souveraineté (II). Les idées développées seront illustrées d'exemples choisis dans le passé mais surtout dans la vie politique du monde contemporain. Une place particulière sera faite à l'évolution possible du statut de la Martinique.

\section{I. - LES ELEMENTS CONSTITUTIFS DU GROUPEMENT «ETAT»}

Ils sont donc au nombre de deux : le territoire et la collectivité humaine installée à demeure sur celui-ci. On remarquera que ces deux éléments en supposent logiquement et humainement un troisième : la possibilité matérielle de vivre sur ce territoire, c'est-à-dire, au moins, de se nourrir, au sens le plus restreint de ce mot, ne pas mourir de faim. Or aucune définition ne tient compte de la question des ressources. Il convient de se demander pourquoi.

Il y a peu à dire concernant le territoire. Il doit exister. Parce qu'ils en sont dépourvus, les vrais peuples nomades, tels les Romani, ne forment pas un Etat. Il n'a pas à être rigoureusement délimité ${ }^{13}$, même s'il est préférable qu'il le soit. Les conflits frontaliers, nombreux à notre époque, ne sont pas du ressort de l'arpenteur mais de l'ONU. Aucun minimum d'étendue n'est imposé.

12. Th. Michalon, «Les sociétés non-industrialisées et l'Etat de droit», in Mélange en hommage à Bernard Vonglis, Paris, 2000, p. 283.

13. Décision du Tribunal arbitral mixte germano-polonais, $1^{\text {er }}$ août 1929 , citée par J.-D. Mouton, op. cit., p. 82. 
Favorisée par la nature, dans ce domaine tout au moins, la Martinique, en tant qu'île, a un territoire parfaitement circonscrit. Pas rares sont les Etats dont la surface est inférieure à ses $1000 \mathrm{~km}^{2}$, dans la Caraïbe en particulier. La Martinique remplit donc sans aucun doute la première condition exigée pour former un Etat.

Il reste en revanche beaucoup à dire concernant le second élément, la collectivité humaine. L'emploi de ce terme imprécis est justifié par le fait qu'il existe à ce propos, dans les textes constitutionnels eux-mêmes, une confusion qu'entretiennent, par manque de rigueur, la plupart des auteurs traitant de cette question ${ }^{14}$.

Les deux premières constitutions françaises écrites, celles de 1791 et de 1793, avaient pourtant bien fixé le sens des mots : la nation, c'est l'ensemble des Français vivants, le peuple, le corps électoral (le populus romain). Malheureusement à tous égards, durant la deuxième moitié du XIX ${ }^{\mathrm{e}}$ siècle, est apparue une conception romantique de la nation qu'expriment, entre autres, Renan et Michelet et que l'on relève encore dans les discours et les écrits de Malraux et du Général De Gaulle. La Nation, communauté de race (!), de langue, de religion (!) ; souvenirs communs ; communauté de rêve d'avenir partagé... D'où l'effort accompli sous la III $^{\mathrm{e}}$ République pour faire disparaître les derniers particularismes locaux, patois et langues régionales que des idées nouvelles remettent de nos jours à l'honneur, parfois comme arguments en faveur d'une indépendance réclamée. Comme les autres pays européens en voie de formation (Allemagne, Italie) faisaient le même rêve, il en est résulté le nationalisme, trois guerres dont deux mondiales, et d'autres horreurs, tel l'holocauste juif.

En prenant l'exemple français, on propose donc de respecter la terminologie suivante :

- Nation, ensemble des Français passés, actuels et à venir ;

- nation, partie de la population du territoire français possédant la nationalité française, ce qui exclut les étrangers admis à résider avec

14. Cf. B. Vonglis, op. cit. 
des droits inférieurs (à Athènes, les Métèques) ainsi que les clandestins (sans papiers).

- Peuple, corps électoral, mais l'ancienneté de la confusion avec l'idée de nationalité ou de religion - les peuples germaniques,

- le peuple juif - fait qu'il est difficile de ne pas s'y soumettre, en dehors du domaine électoral proprement dit.

Si le terme de nation, avec ou sans majuscule, inspire la peur en raison des mauvais souvenirs qu'il rappelle, il n'en demeure pas moins qu'une partie de son contenu est encore considérée par les théoriciens comme indispensable à l'existence d'un Etat. Pour que la population d'un territoire justifie celle-ci encore faut-il que règne entre ses membres une certaine cohésion, garante de la survie de l'entité politique. Sinon une «volonté de vivre ensemble», du moins l'acceptation de ce fait.

Dans la réalité ce minimum n'existe pas toujours, en dehors même du cas aberrant de l'Ordre de Malte, doté d'un territoire et de la souveraineté, mais dont les membres conservent leur nationalité d'origine, ce qui ne l'empêche pas d'être un Etat reconnu par 83 pays. Il existe aussi depuis peu des cyberétats, dont certains localisés sur des îles imaginaires qui n'ont d'existence que sur l'Internet ce qui n'a pas empêché le chef de l'un d'eux d'entrer aux îles Fidji sur présentation de son passeport... cybernational.

Le nombre des habitants n'est pas en cause. Comme pour le territoire, aucun minimum n'est exigé. Très logiquement, Bodin le fixait à trois ménages, les chefs de deux d'entre eux constituant la majorité indispensable pour gouverner les trois ${ }^{15}$.

Les vraies difficultés ressortent d'un examen de la situation dans le monde actuel, où les deux événements majeurs de la seconde moitié $\mathrm{du} \mathrm{XX}^{\mathrm{e}}$ siècle, décolonisation et décommunisation, ont eu des conséquences graves, voire dramatiques.

15. J. Bodin, op. cit., L. $1^{\mathrm{er}}$, ch. 2. 
En Afrique particulièrement l'ONU a reconnu la qualité d'Etat à de nombreux pays colonisés sans se soucier de l'existence d'un minimum d'unité ethnique et culturelle au sein de leur population. Deux motifs honorables à ce laxisme : l'urgence de la décolonisation, l'idée que le cadre de l'Etat faciliterait l'unification. On les appelle des quasi-Etats ou Etats de l'ONU. Les conséquences néfastes de cette hâte doublée d'une illusion ne se sont pas fait attendre et subsistent toujours. Les clivages ethniques s'étant révélés plus forts que l'autorité de l'Etat, il en est résulté un mépris des frontières (touaregs et Mali), des massacres interethniques (Congo Kinshasa, Rwanda...) qui ont entraîné des ingérences étrangères restreignant d'autant la souveraineté de ces pays.

En Europe, la décommunisation a conduit à l'éclatement d'Etats jusqu'ici maintenus artificiellement en vie par un pouvoir autoritaire. La Russie a dû admettre l'indépendance de Républiques dominées ou d'anciens Etats indépendants conquis par elle, tels les pays baltes. L'actuelle répression en Tchétchénie a, outre des buts politiciens inavoués, celui de donner un coup d'arrêt à ce processus de désintégration. Quant à la Yougoslavie, Etat composite né de la première guerre mondiale, elle a subi un véritable éclatement. Dans les deux cas s'est posé le problème des minorités, minorité russe dans les pays baltes, minorités religieuses et ethniques dans l'ancienne Yougoslavie où une barbare politique de «purification » a conduit aux massacres de Bosnie et du Kosovo. L'OTAN a profité de son intervention pour imposer son contrôle dans cette partie de l'Europe. Gageons qu'elle n'est pas près d'y renoncer.

En Tchécoslovaquie, autre Etat né de la première guerre mondiale, la séparation des deux peuples s'est faite d'un commun accord. A l'inverse, l'Allemagne s'est réunifiée sous le regard inquiet de ses anciens ennemis européens. Mais une nation divisée subsiste, la Macédoine.

Indépendamment de la décommunisation, on rappellera le cas du peuple Kurde, écartelé entre trois Etats. Celui de l'Irlande du nord où la paix paraît bien difficile à rétablir. Celui de la Belgique, autre Etat composite né lui, de la révolution de 1830 , où coexistent difficilement deux peuples et deux langues. 
En France, si un passé vieux de plus d'un millénaire permet d'affirmer qu'il existe une Nation, force est bien de reconnaître qu'il n'y a plus de nation, c'est-à-dire de réelle volonté de vivre ensemble et non pas seulement l'un à côté de l'autre - de faire passer l'intérêt général avant les intérêts particuliers et sectoriels. Plus de patriotisme ce qui n'est peut-être pas à regretter compte tenu des désastres que ce sentiment, partagé par d'autres peuples, a provoqué - ni, malheureusement, de civisme. Pourtant, sauf pendant la Révolution, on n'a jamais autant employé le mot «citoyen », singulièrement sous forme adjective, ce qui est nouveau.

Un dossier documentaire du Monde, d'avril 2000, intitulé La citoyenneté entre crise et utopie énumère les causes de ce déclin : l'incapacité de l'Etat à gouverner et à faire régner l'ordre, les «affaires », l'affaiblissement des syndicats, le chômage, l'incapacité de l'enseignement à assurer l'égalité et l'intégration des minorités ethniques.

Les Martiniquais forment-ils une nation ? A cette question Aimé Césaire a dès longtemps répondu par l'affirmative. Telle est également l'opinion d'Alfred Marie-Jeanne présentée par Jeanne Yang-Ting ${ }^{16}$. Cette nation existe depuis l'abolition de l'esclavage en 1848 et les Martiniquais en sont conscients depuis les émeutes foyalaises de décembre 1959. C'est sur ce fondement qu'A. Marie-Jeanne souhaite voir l'ONU reconnaître le droit de l'île à l'autodétermination conduisant à l'indépendance (Résolution 1514 de l'AG, 14 déc. 1960 : «... tous les peuples coloniaux ont le droit de libre détermination... »). Or si la Martinique est citée sur la Liste des territoires non autonomes (Résol. 66 de l'AG, 1946) elle ne figure pas sur celle établie en 1990. Donc l'ONU est maintenant d'accord avec la France. C'est qu'entre temps la départementalisation, demandée par les élites martiniquaises de couleur pour lutter contre la domination des Blancs créoles, puis l'accession à la parité, réclamée à conques et à cris pendant 40 ans, ont transformé l'ancienne colonie en un département comme les autres, au moins en ce qui concerne le niveau de vie et la protection sociale. A

16. J. Yang-Ting, Le mouvement indépendantiste martiniquais, présentation $d u$ Marie-Jeannisme, Petit-Bourg (Guadeloupe), 2000, p. 65-75. 
quoi s'ajoutent des avantages salariaux (les $40 \%$ des fonctionnaires) et fiscaux (réduction de $30 \%$ plafonnée à 33000 francs). Le tout avec un inconvénient inévitable à cette époque : la centralisation, depuis peu nettement réduite.

Oui, le peuple martiniquais, toutes couleurs de peau mêlées, forme une nation. Une nation qui n'est plus colonisée mais intégrée à une nation plus vaste, la nation française ${ }^{17}$. C'est en effet une société spécifique, qui s'est constituée au fil des siècles par la « collaboration »d'européens qui venaient chercher fortune aux îles et d'africains qui y ont tous été amenés de force et contraints pour la plupart à y rester. Après 1848 sont arrivés des Indiens, les Coolies, terme qui révèle bien ce qu'on en attendait et le cas qu' on en faisait ${ }^{18}$. Mais le melting pot n'a pas fonctionné parfaitement ${ }^{19}$. Des antagonismes multiples, interethniques et intraethniques existent. La formation d'une élite intellectuelle et économique de couleur, les mulâtres, est venue combiner les trois clivages, l'ethnique,

17. De même qu'il existe un peuple ou une nation corse, en dépit de la dénégation implicitement contenue dans la constitution de 1958 et explicitée par le Conseil Constitutionnel, mais au sein de la nation française. On peut également se poser la question pour des provinces françaises rattachées tardivement au royaume et qui ont plus ou moins conservé leurs particularismes locaux, telles la Bretagne et l'Alsace. Il existe incontestablement un peuple basque dont la langue a une origine inconnue. La réponse doit être laissée aux intéressés, mais dans le calme et dans le cadre de la nation française. Pour un historique d'idée de nation martiniquaise, voir E. Jos, «Identité culturelle et identité politique, le cas martiniquais », in Cinquante ans de départementalisation outre-mer, ss la dir. de F. Constant et J. Daniel, Paris, 1997, p. 338-353.

18. Pour avoir une idée du mépris dans lequel étaient tenus les Coolies à la fin du $\mathrm{XIX}^{\mathrm{e}}$ siècle, voir A.-Ph. Blerald, « La citoyenneté française aux Antilles et ses paradoxes », in Cinquante ans...., op. cit., p. 195-196.

19. Contrairement à ce qu'on entend dire parfois, il n'a guère mieux fonctionné à Maurice et à la Réunion où le rapport de mépris est inversé. Là, ce sont les originaires d'Afrique, les Cafres, qui sont considérés comme des inférieurs. Les travaux pénibles leur sont en majorité réservés. Commerce et fonction publique sont monopolisés par les Malbars. Le monument érigé à Saint-Denis il y a une dizaine d'années pour commémorer l'abolition de l'esclavage était souillé d'inscriptions racistes. Elles ont été effacées en juillet 2000. La presse locale fait état des revendications des Cafres. Et bien sûr, comme à la Martinique, les inscriptions «Fwancé déor » ne manquent pas, même si l'adverbe est orthographié un peu différemment. 
l'économique, le social ${ }^{20}$. Pourtant, les facteurs d'unité ne manquent pas : le créole, le style de vie... et la francisation très poussée. Même si le groupe des Blancs créoles et celui des Martiniquais de couleur sont relativement différenciés par les souvenirs du passé esclavagiste et le clivage économique - les premiers disposent de $90 \%$ des terres et contrôlent largement la grande distribution - il n'existe pas en Martinique d'opposition violente entre groupes ethniques, comme c'est le cas dans certains Etats africains (Hutus et Tutsis au Rwanda), ni scission linguistique comme en Belgique, ni scission religieuse comme dans l'ancienne Yougoslavie ou en Irlande du nord.

Qu'adviendrait-il de cette unité et de cette paix sociale relatives, dues à la conscience que chacun a de ce qu'il peut ou non faire dans le cadre départemental actuel, si la Martinique accédait à l'indépendance ? Tout dépendrait en fait des conditions dans lesquelles s'organiserait le pouvoir. Il est évident que si le MIM domine la situation, ce ne sont pas seulement les Blancs créoles - dont A. MarieJeanne prévoit la confiscation des terres - et les Métropolitains qui partiront, mais aussi une fraction de l'élite autonomiste qui a décidé le moratoire de 1981. Ceux qui resteront, désireux de vivre ensemble ou plus ou moins contraints d'adhérer au nouveau pacte social, formeront bien encore une nation. Si au contraire, sous l'égide d'hommes politiques moins dogmatiques et plus modérés, l'indépendance conduisait à des concessions réciproques, la société martiniquaise actuelle pourrait subsister, riche de la diversité de ses composantes.

Pour mieux faire face aux problèmes économiques que devrait affronter le jeune Etat martiniquais.

Car il importe de s'intéresser à une question essentielle, pourtant habituellement tenue à l'écart du débat général, celle des ressources.

20. Sur cette question, voir J.-Cl. William, Compère lapin et compère mulet : métissage et comportement socio-politique à la Martinique, thèse sc. pol. multigr., Paris IX, 1988 ; «Réflexions sur la périodisation de l'histoire de la Martinique », in Mélanges... Vonglis, op. cit., p. 363-372. 
Celles-ci dépendent à la fois de l'étendue et de la richesse du territoire, ainsi que de la densité de la population qui y vit.

Le point de vue de l'ONU à cet égard est clairement énoncé dans la résolution 1514: «Le manque de préparation dans les domaines politique, économique, social... ne doit pas être pris comme prétexte pour retarder l'indépendance». Le mot «prétexte » en dit long : c'est une référence au traité de Berlin de 1885, qui fondait au contraire sur ce motif la nécessité de la colonisation. La résolution 2592 confirmera la 1514 en déclarant que l'indépendance ne doit pas être retardée par «... les questions d'isolement géographique et de ressources limitées... ${ }^{21}$. Point de vue généreux, qui visait à hâter cette décolonisation considérée comme le bien absolu, même si les hommes ne peuvent pas vivre seulement d'indépendance et d'eau fraîche, dont ils ne disposent d'ailleurs pas toujours.

Le résultat ne s'est pas fait attendre. Le dramatique manque de ressources de certains pays décolonisés - ou décommunisés - les a conduits à une dépendance totale à l'égard du FMI ou de la BM, mais aussi à des fraudes et gâchis colossaux (Côte d'Ivoire, Russie). Sans parler du chantage que pratiquent certains anciens pays soviétiques, comme l'Ukraine avec la mise hors service de la centrale atomique de Tchernobyl. Ces pays font payer à l'Occident la faillite prévisible et dès longtemps dénoncée d'un système économique absurde.

La dépendance à l'égard des instances financières internationales a entraîné dans ces pays la dégradation du pouvoir. On constate celle-ci en Russie où les mafias ont pris en main l'économie, à leur unique profit. La volonté d'indépendance des Tchétchènes ne repose pas seulement sur des raisons ethniques et religieuses. En Afrique, la conception patrimoniale du pouvoir, les liens familiaux, le clientélisme, la domination d'une ethnie sur les autres provoquent des abus, des accaparements qui privent de nourriture une partie de la population et ruinent l'agriculture. Les conséquences en sont parfois si

21. Voir A. Le Sausse, Que faire pour développer la Martinique?, Fort-de-France, 1974 ; F. Célimène et P. Watson, Economie politique caribéenne, Paris, 1991. 
graves que les pays occidentaux ont proclamé et mis en œuvre le droit d'ingérence. Que reste-t-il alors de la souveraineté de ces Etats ? Certains pays, en Amérique du sud, en Extrême-Orient, compensent leurs défaillances économiques par la production et l'exportation de stupéfiants. Sait-on que le chiffre d'affaires global ainsi réalisé est supérieur à celui du pétrole? Même dans un pays développé, l'insuffisance de l'économie d'une partie du territoire risque de provoquer le démembrement de l'Etat: certains, en Italie du Nord, région prospère, envisagent de se libérer du boulet, mafieux au surplus, qu'est le sud de la botte.

Certes, aucun Etat ne peut vivre en complète autarcie, ou très mal, lorsqu'il y est contraint (Irak, Cuba). Les pays riches eux-mêmes doivent importer, le pétrole en particulier. Depuis 1973, les trois chocs pétroliers successifs ont provoqué récession, inflation, chômage et guerres. Les pays riches ont également besoin d'exporter. Enfin, la prospérité économique confère du pouvoir sur les autres Etats. Les USA et, à un moindre degré, le Japon et l'Allemagne en sont des exemples.

Concluons: un pays qui, en raison de l'aridité de son sol, l'absence dans celui-ci de ressources échangeables contre des produits manufacturés et de la nourriture, ou qui, disposant de quelques ressources, mais surpeuplé, ne peut fournir à sa population le minimum vital, ne saurait être considéré comme un Etat, cette notion supposant nécessairement l'indépendance, l'une des manifestations concrètes de la souveraineté. C'est un quasi-Etat qui ne survit que grâce à la sollicitude non désintéressée des pays riches. Et ce ne peut être en aucun cas un Etat de droit, même si l'ordre y règne, comme en Irak ou à Cuba. A partir de quel degré d'auto-suffisance un pays sousdéveloppé peut-il être considéré comme un Etat ? Il n’y a évidemment pas de réponse chiffrée à cette question. Tout est affaire de cas particuliers. Cuba est un Etat, la Somalie, non. C'est la raison pour laquelle il n'est pas possible d'introduire dans la définition de l'Etat donnée ci-dessus une expression concrétisant l'exigence d'un minimum de ressources. Mais celle-ci est, comme on l'a déjà laissé entendre, implicitement contenue dans l'impératif de souveraineté. 
Et la Martinique ? Force est bien de reconnaître qu'elle est de ce point de vue très défavorisée, comme le reconnaissent les spécialistes $^{21}$. Par sa position géographique d'abord qui la tient à l'écart des grands courants d'échanges ${ }^{22}$, celui de la drogue excepté. Par sa vocation à produire du sucre et de la banane invendables sans subventions, en raison de leur prix de revient non concurrentiel. Par le haut niveau des salaires par rapport aux pays environnants, qui rendrait non compétitif à peu près tout ce qui pourrait y être fabriqué. Par le niveau de vie élevé de l'ensemble des Martiniquais, les habitudes de consommation prises au fil des ans.

Enfin, la Martinique souffre de l'absence de tout plan crédible c'est-à-dire réaliste et concret - de prise en main de la vie économique de l'île devenue indépendante, et singulièrement de la part des autonomistes et indépendantistes qui ont eu pourtant quatre décennies pour y penser. Ils en sont encore au stade de la réflexion sur ce sujet capital, comme le reconnaît Serge Baret $^{23}$. Le programme de développement présenté par le PPM en 1970 - lutte contre le chômage par une politique de grands travaux, l'exploitation de la terre et des richesses de la mer, réduction ou partage du temps de travail, soutien public aux petites et moyennes entreprises - est d'une évidence troublante. Les buts sont fixés. Mais quid de la méthode et des moyens? A propos de l'exemple de la pêche, E. Mariette montre clairement les obstacles intérieurs et extérieurs auxquels se heurte toute tentative en ce domaine ${ }^{24}$.

22. Les échanges régionaux, que ce soit avec la Guadeloupe ou les îles et Etats continentaux de la Caraïbe sont minimes. Les productions de ces pays sont en effet concurrentes et non pas complémentaires.

23. Dans sa conférence du 23 mars 2000, Serge Baret déclarait qu'une commission de réflexion créée après l'arrivée d'A. Marie-Jeanne à la présidence du Conseil régional avait préparé en tout et pour tout deux projets : le développement de l'élevage des écrevisses et la création de celui des poissons d'aquarium.

24. J. Mariette, La sociale démocratie locale, modèle politique pour la Guadeloupe et la Martinique?, Petit-Bourg (Guadeloupe), 2000, p. 183-184. En dépit du soutien financier du Conseil régional et du programme de développement mis en place par les autorités communautaires, la pêche martiniquaise ne couvre que $40 \%$ de la consommation. 
La situation économique actuelle de la Martinique est clairement posée par J. Mariette: il y a un demi-siècle, l'île était pauvre et exportait beaucoup ; devenue riche, elle n'exporte que très peu. Le problème est donc double. Il faut, d'une part, augmenter la production et, d'autre part, trouver le moyen de la vendre à l'extérieur. Augmenter la production de sucre ? Oui, à la condition d'investir largement. Augmenter celle des bananes ? Oui, mais sous la réserve, également valable pour le sucre, de réduire les coûts de production, c'est-à-dire de diminuer les salaires. Les deux filières ne subsistent que grâce aux subventions européennes que réprouve l'OMC. Reste la production de fruits, légumes et viandes. Dans ce domaine, le développement est possible, à la condition de reconvertir des terres consacrées à la banane et au sucre, ou de pratiquer la culture et l'élevage hors sol comme le propose J. Mariette ${ }^{25}$ dont le programme social-démocrate accepte comme tel le quasi-monopole des terres exercé par les Blancs créoles. Reste aussi l'aquaculture qui peut être largement développée. Non seulement la Martinique deviendrait ainsi autosuffisante, mais elle pourrait même exporter à destination des nombreux Antillais vivant en Métropole et susceptibles de constituer ce que notre collègue Fred Célimène qualifie de «marché ethnique ${ }^{26}$.

La réduction du niveau de vie des Martiniquais est donc inévitable dans la perspective d'une autonomie destinée à préparer sérieusement une indépendance ultérieure, a fortiori si celle-ci était proclamée à court terme, sans préparation. A ce propos, l'alternative est claire: ou subir ce recul comme une punition - ce serait la conséquence inévitable d'une soviétisation du pays - ou l'accepter, la créer volontairement et l'affronter avec civisme. La deuxième solution est préconisée par Thierry Michalon. Notre collègue propose de laisser le choix à une assemblée locale dotée du pouvoir de renverser son exécutif. Cette assemblée prendrait la décision de réduire les rémunérations, celles des fonctionnaires en particulier. Quant à la production locale, il suggère de la développer progressivement, secteur

25. Op. cit., p. 179.

26. F. Célimène et $\mathrm{P}$. Watson, op. cit., « :... le soi-disant marché « ethnique » dont la base est la communauté d'origine africaine caribéenne et asiatique vivant à l'étranger ». 
par secteur. Un produit manufacturé serait fabriqué sur place et inévitablement vendu à un prix supérieur que, par civisme, les Martiniquais accepteraient de payer. Deux pistes sérieuses à suivre pour responsabiliser les élus et redresser une situation économique bien compromise.

Un projet modeste, certes. Il en existe de plus ambitieux. C'était déjà le cas de celui proposé en 1974 par Alex Le Sausse ${ }^{27}$. Il repose sur un principe formulé en conclusion : «plus un pays est petit, plus ses ressources sont réduites, et plus il devra compter sur ses exportations pour satisfaire ses besoins et plus son développement en dépendra ». Pour parvenir à ce résultat, la Martinique devra s'intégrer à l'Europe qui lui accordera un protectionnisme temporaire. Elle diversifiera son agriculture ${ }^{28}$ pour satisfaire la demande locale et exporter. A l'usage du marché local ou intra-régional, elle développera une chaîne agro-alimentaire et créera des usines de petit équipement. A destination du marché commun, des produits de haute qualité et de prix élevé. Et surtout, on développera le tourisme. De leur côté, Fred Célimène et Patrick Watson ${ }^{29}$, après avoir constaté, dans la Caraïbe, l'échec des politiques économiques unilatérales - industrialisation sur invitation, par l'installation d'entreprises métropolitaines ; interventionnisme étatique - et du mouvement d'intégration régionale, avec la CARICOM depuis 1973, proposent, non sans un certain pessimisme $^{30}$ quant aux résultats, de s'intéresser à des produits

27. Op. cit., passim.

28. Diversifier l'agriculture martiniquaise, c'est un vœu pieux depuis la 2 moitié du XVII ${ }^{\mathrm{e}}$ siècle ou les marchés européens du tabac et du sucre furent successivement et temporairement saturés. Après l'échec de l'indigo et du mûrier, on s'intéressa au cacao, jusqu'en 1727 où un tremblement de terre détruisit la plupart des cacaoyères, puis au café, qui connut son apogée pendant les années 1770. Mais la canne demeura la production de base, au $\mathrm{XIX}^{\mathrm{e}}$ siècle encore. La production de racines et de bananes destinées à la nourriture des esclaves à toujours été insuffisante au XVIII ${ }^{\mathrm{e}}$ siècle, en dépit de statistiques optimistes reposant probablement sur de fausses déclarations des habitants. Sur ces questions, voir P.F.R. Dessalles, Les Annales du Conseil souverain de la Martinique, 4 t., dont 2 de notes, par B. Vonglis, Paris, 1995.

29. Op. cit., passim. 
nouveaux en assimilant les technologies les plus récentes; de réduire les coûts par une politique salariale dont les auteurs prévoient qu'elle se heurtera à la résistance des travailleurs ; de développer le tourisme, moteur de la croissance.

Un mot vient à l'esprit à la lecture de ces projets : volontarisme. Les Antilles ne sortiront de leur ornière économique et sociale que grâce à l'effort de tous, grâce aux sacrifices que devront consentir les moins défavorisés. Le projet le plus remarquable de ce point de vue est celui d'Ernest Moutoussamy ${ }^{31}$. L'auteur part d'un constat indiscutable: en système d'économie libérale, la finalité des entreprises privées n'est pas de créer des emplois mais d'être compétitives sur le marché. Il propose donc d'introduire «un peu de marxisme » pour redynamiser la production locale : agriculture, agroalimentaire, tourisme, industrie (fabrication d'équipements ménagers portant le label «made in DOM », pour changer les comportements des consommateurs). Mais on se gardera de diminuer les salaires pour compenser on maîtrisera le coût des transports -, et on garantira aux producteurs de canne un revenu minimum. On établira, outre l'assemblée unique, une agence départementale de travail non marchand qui mènera une politique de formation pour favoriser l'insertion de la jeunesse oisive et délinquante, que l'on initiera également aux sports. Il sera donc nécessaires de créer des milliers d'emplois d'animateurs professionnels et sportifs, plus 5 à 7000 postes dans l'enseignement. Programme intéressant, on en conviendra, mais qui paiera ? L'Union européenne, en créant au profit des Antilles un statut d'ultrapériphéricité, et, bien sûr, l'Etat, mais sous une forme qui ne devrait pas lui coûter plus qu'il ne verse actuellement. Partant de la constatation que les sans-emploi antillais, tout comme les métropolitains, préfèrent au SMIC la combinaison « RMI + Djob », la France affectera à ces missions les fonds du RMI, des CES à quoi s'ajouteront des prélèvements sur les allocations familiales, sur les pensions, sur le rapport des jeux. Enfin l'auteur formule une exigence

30. Pessimiste également J. Nosel, «Appréciation de l'impact économique de la départementalisation à la Martinique », in 1946-1996, Cinquante ans..., op. cit., p. 6667.

31. E. Moutoussamy, Inventer l'emploi en Outre-mer, Paris, 1997. 
que l'on ne peut qu'approuver : l'application rigoureuse de l'Etat de droit $^{32}$.

Tous ces plans supposent une mutation plus ou moins drastique des habitudes et des mentalités locales, un véritable sursaut d'énergie et de civisme. Il reste à en tenter la réalisation. Mais qui l'osera?

Il n'y a donc pas d'Etat, en tout cas d'Etat digne de ce nom, sans territoire, sans population homogène, sans un minimum raisonnable de ressources. Soit sans domaine d'exercice ni titulaire(s) de la souveraineté au nom de laquelle l'Etat gouverne et représente UN Etat.

\section{II. - LE POUVOIR CONFERE AU GROUPEMENT « ETAT» : LA SOUVERAINETE}

Juristes, politistes et philosophes ont tant glosé sur cette notion, elle est devenue si extensive et floue, qu'il est indispensable de commencer par la définir ${ }^{33}$.

32. Sur cette question, voir A.-M. Le Pourhiet, La perception du droit à la Martinique, ibid., in fine. «Pourquoi la société martiniquaise a-t-elle tant de mal à assimiler l'Etat de droit ? » demande l'auteur à qui, dans son introduction, p. 22, notre collègue Justin Daniel reproche de récuser « a priori toute tension entre l'universalisme de la règle de droit et les particularismes culturels ». La réponse étant ainsi donnée au «pourquoi ? », il reste à préciser les conséquences pratiques de cette tension. Faut-il admettre qu'il y ait des sociétés développées où il soit définitivement impossible de faire respecter le droit communément admis, en matière de circulation par exemple, compte tenu de l'intérêt bien compris des fraudeurs eux-mêmes? Quant au comportement parfois répréhensible de ceux qui sont chargés du maintien de l'ordre, il constitue l'un des obstacles majeurs à l'effectivité d'un Etat de droit dont les pays démocratiques se réclament en principe. Qu'on se rappelle l'affaire aussi cocasse que scandaleuse de la vedette de la gendarmerie de Guadeloupe, chargée de cadeaux de Noël achetés hors taxes à Saint-Martin, que les douaniers ont saisie par vengeance alors que d'habitude ils fermaient les yeux sur cette fraude.

33. Sur la notion de souveraineté, voir B. Vonglis, « L'Etat, c'était bien lui», Essai sur la monarchie absolue, Paris, 1997, p. 60-70 et la bibliographie citée. 
La souveraineté est une notion française. Sa définition et ses rapports avec son ou ses titulaires ont été établis, au tournant des $\mathrm{XVI}^{\mathrm{e}}$ et XVII ${ }^{\mathrm{e}}$ siècles, par trois juristes : Jean Bodin, Charles Loyseau et Cardin Le Bret. Pour le premier, c'est «... le pouvoir de donner loi... sans le consentement de plus grand, ni de pareil, ni de moindre que soi » ${ }^{34}$ et tous les autres pouvoirs découlent de là. Elle est inaliénable et non délégable. Elle est aussi impartageable : « la souveraineté n'est non plus divisible que le point en la géométrie » (Le Bret $)^{35}$. On peut conclure que la souveraineté consiste dans la réunion de tous les pouvoirs entre les mains d'une même personne physique ou morale. La souveraineté appartient à l'Etat. Ils sont inséparables. Pour Loyseau, elle est « la forme qui donne l'être à l'Etat ${ }^{36}$.

Dans un but pratique uniquement, car l'un postule l'autre, on peut distinguer deux aspects de la souveraineté : la souveraineté externe et la souveraineté interne. La première, négative, soustrait UN Etat à toute intervention étrangère ; c'est l'indépendance nationale, expression qu'il serait préférable de substituer à celle de souveraineté externe, puisque celle-ci est nécessairement limitée par celle des autres Etats, ce qui est incompatible avec la notion de souveraineté. La seconde, positive, est l'ensemble des pouvoirs que détient le monarque au niveau suprême - il en délègue une partie à des agents en se réservant le dernier ressort - ou le peuple (corps électoral) qui les exerce directement ou par l'intermédiaire de représentants élus. Monarque, peuple, représentants, ainsi que leurs agents, forment L'Etat. Dans le dernier cas, tant que durent les fonctions des représentants le peuple ne conserve de sa souveraineté que le pouvoir constituant, sous réserve, dans la constitution de la $V^{\mathfrak{e}}$ République, que le président décide de le consulter. C'est le référendum bonapartiste. La prétendue souveraineté du peuple est donc un leurre. Seul le référendum d'initiative populaire lui permettrait de la mettre en œuvre.

34. J. Bodin, op. cit., L. $1^{\mathrm{er}}$, ch. 10, Première marque de souveraineté.

35. C. Le Bret, De la souveraineté du Roy, Paris, 1632.

36. Ch. Loyseau, Des seigneuries, Paris, 1613, III, 5. 
Longtemps cette théorie de la souveraineté correspondit à la réalité, en France en tout cas, quoique plus en plus difficilement après 1789. Mais, en cette fin du $\mathrm{XX}^{\mathrm{e}}$ siècle, les souverainetés nationales souffrent de telles atteintes que la notion, et par conséquent celle d'Etat, deviennent, pour des raisons différentes, inadéquates à la réalité politique française comme à la réalité politique mondiale.

Pour la France, la question ne présente plus d'intérêt que pour les «souverainistes» de tous bords, soutenus par une partie non négligeable, mais minoritaire, des citoyens. Le TUE (Maastricht, 1992) a tué la souveraineté de la France en la soumettant à une législation et à une justice supérieures aux siennes. Elle n'est donc plus vraiment un Etat, même si elle en présente encore majoritairement les caractéristiques. L'acceptation de l'Euro - qu'Anglais et Danois ont eu la bonne idée de refuser - a sonné le glas de l'indépendance française en la privant d'un droit régalien. Certes, depuis longtemps déjà, la France n'était plus entièrement indépendante dans le domaine financier. Elle devait par exemple faire varier ses taux d'intérêts en fonction de ceux de la Bundesbank. Mais légiférer en s'adaptant au milieu économique n'est pas légiférer sur l'ordre d'un pays étranger. Au contraire, renoncer au franc, subir des condamnations de la part de la Cour de Justice des Communautés Européennes, recevoir des ordres de la $\mathrm{CE}$ - accepter d'importer du bœuf anglais, faire rembourser par les industriels français les aides qu'ils ont reçues du gouvernement, etc... - c'est perdre son pouvoir de légiférer et de gouverner sans contrainte extérieure et, conséquemment, sa qualité d'Etat.

En 1992, le Garde des sceaux déclarait encore, selon la doctrine traditionnelle, que la souveraineté ne peut être ni aliénée ni transférée $^{37}$. Quelle conclusion en a tirée le gouvernement d'alors ? Que la France devait sortir de l'Union Economique et Monétaire ? Non, il a fait réviser l'art. 88.2 de la Constitution, qualifiant la perte d'un droit régalien de «transfert de compétences » ${ }^{38}$. Toujours la

37. JO. AN. CR., 6 mai 1992, p. 845.

38. $L C \mathrm{n}^{\circ} 92-554$ du 25 juin 1992, art. 5 . 
rhétorique, la magie du verbe, l'innocence des mots, pour masquer une réalité cruelle. Dans le même but, on recourt à des arguties juridiques, autre tendance bien française: il n'y aurait pas aliénation mais seulement délégation d'éléments de souveraineté. Or, si le pouvoir peut être délégué, si un élément de celui-ci, une «marque » de souveraineté (J. Bodin), peut l'être, c'est seulement sous réserve que la délégation puisse être reprise, ce qui n'est pas le cas.

Une question se pose d'ailleurs à ce propos : qui est souverain en Europe communautaire puisque celle-ci ne l'est pas encore ? Si, comme il est probable, elle devient fédérale, toute souveraineté disparaîtra sur le territoire qu'elle recouvre, car, notre collègue Olivier Beaud l'a démontré, cette notion est incompatible avec ce type de constitution $^{39}$. Cette particularité n'empêchera pas l'Europe de fonctionner, comme le montre l'exemple des Etats-Unis, mais il n'en demeurera pas moins que la France aura perdu sa souveraineté.

Mais il y a plus grave : l'Etat français se vide de sa souveraineté non seulement par le haut, au profit des instances européennes, mais aussi par le bas, avec le cours qu'est en train de prendre la décentralisation. Les aspirations autonomistes, voire indépendantistes, de la Corse et des DOM ne sont plus condamnées mais seulement différées. Le Président de la République, garant du respect de la constitution et de l'intégrité du territoire, ainsi que le Premier ministre paraissent bien d'accord sur ce point, même si leurs déclarations comportent des réserves qui ne sont guère respectées en Corse. Calqué sur le modèle calédonien, le projet de statut pour celle-ci (accords de Matignon) lui reconnaît un pouvoir législatif, d'abord sous le contrôle du gouvernement, puis sans contrôle. La première solution serait acceptable en principe puisque l'Ancien Régime, en dépit de l'absolutisme, reconnaissait ce pouvoir aux Parlements. Ceux-ci l'exerçaient par le moyen d'arrêts de règlement susceptibles de cassation par le conseil du roi. Mais la seconde étape consacrera un abandon de souveraineté et donc l'indépendance de l'île. Les avantages promis à la Corse et aux DOM encouragent déjà les revendications de provinces de l'Hexagone: Pays basque, Bretagne, Alsace, Savoie...

39. O. Beaud, «Fédéralisme et souveraineté », RDP, 1998, 1, p. 83-122. 
Cette évolution est souhaitée par certains leaders politiques à la recherche d'une occasion de rebondir, tel François Léotard, ou désireux de prendre date au sein de l'opposition et d'influencer celleci, comme Alain Madelin. Au niveau européen elle est préparée par le Conseil des Régions, qui fonctionne difficilement parce que les Etats s'efforcent de le museler. Mais les Länder allemands exercent des pressions dans ce sens ${ }^{40}$. Il paraît vraisemblable qu'en France - en retard d'une décentralisation comme elle le fut longtemps d'une guerre - ce mouvement se transformera en débâcle pour des régions encore peu habituées à se gouverner elles-mêmes, alors qu'il se réalisera aisément en Belgique, en Italie, en Espagne. C'en sera fini du grand œuvre de ces rois qui firent la France ! Le fait que les DOM aient des représentations auprès des institutions communautaires n'est-il pas une invitation à sauter dès que possible l'échelon intermédiaire de l'Etat ?

Affaire à suivre... Mais il est très probable que, décevant les espoirs de Claude Lise $^{41}$, la centralisation européenne remplace la centralisation étatique ${ }^{42}$. L'Europe en aura besoin pour s'affirmer comme jadis les rois de France. On pourra parler basque, breton, créole... mais il faudra, au nom de la rationalisation de la production et du commerce, respecter le diamètre des concombres fixé par les technocrates bruxellois, les fromages au lait cru seront interdits pour des raisons sanitaires, les combats de coqs et les corridas également au nom de l'écologie... Les Länder allemands, qui sentent venir le vent

40. I. Smets, «Le Comité des régions : une réforme en trompe l'œil ?», in De Maastricht à Amsterdam..., op. cit., p. 127-131.

41. Espoirs exprimés dans sa conférence du 24 février 2000. Claude Lise est président du Conseil général de la Martinique.

42. Voir S. Marti, in Le Monde, Economie, 12 sept. 2000 : L'autonomie régionale a-t-elle un avenir économique ? «La commission de Bruxelles et les Etats recentralisent en douceur... L'Etat-nation a encore de beaux jours devant lui... Le traité de Maastricht, à travers la recentralisation à Bruxelles et Francfort d'importantes prérogatives nationales est plutôt défavorable à l'indépendance des régions ». 
essaient d'y résister en préconisait un deuxième niveau de subsidiaritét ${ }^{45}$.

La notion de souveraineté est également inadéquate à la réalité politique de nombreux « Etats » dans le monde.

Comme on l'a vu plus haut, les Etats de l'ONU ou quasi-Etats subissent l'ingérence justifiée du FMI et de la BM. Ces organismes leur imposent, mais aussi à l'Indonésie, au Brésil et à la Russie, des réformes libérales dont la liste est qualifiée de «consensus de Washington ». Ces pays ne sont plus souverains même si, il y a peu encore, Boris Eltsine soutenait le contraire en rappelant au président Clinton que la Russie dispose encore de nombreuses fusées nucléaires prêtes à partir.

Quant à l'hétérogénéité de la population, elle provoque le démantèlement de l'Etat. Clans et tribus sont séparés par des frontières internes et s'arrogent des pouvoirs qui, sans leur attribuer la souveraineté, ruinent celle de l'Etat. Celui-ci survit néanmoins par application du principe de continuité qui rend très difficile la perte de la qualité d'Etat, même en cas d'anarchie totale. La Somalie est si parfaitement l'illustration de cette réalité qu'elle a eu le triste honneur de donner son nom à ce processus d'implosion de l'Etat: la somalisation est l'étape qui précède la balkanisation.

Le manque d'homogénéité ethnique entraîne normalement la domination d'une ethnie qui se maintient au pouvoir par la force et la corruption. Mais ces pratiques n'épargnent pas les autres Etats non industrialisés. D'où l'exigence d'un personnel politique désintéressé et respectueux du droit, de juges impartiaux, de fonctionnaires compétents et gratifiés de traitements réguliers et suffisants... Mais un pays qui remplit ces conditions est déjà un pays développé.

43. I. Smets, op. cit., p. 129 : «Les Lander sont arrivés à la conclusion que leurs intérêts pouvaient être mieux défendus à travers les canaux nationaux de participation à la prise de décision européenne ». 
La conséquence de cette faillite de l'Etat est le dédoublement de la souveraineté. Privés de souveraineté interne, les quasi-Etats conservent une souveraineté externe limitée au droit de non-ingérence qui interdit aux autres Etats d'intervenir dans leur gouvernement. $\mathrm{Ce}$ droit n'est effectif que dans la mesure où l'ONU en garantit le respect, sous réserve d'exceptions qu'elle cautionne (Somalie, Kosovo). On parle même dans ce cas de devoir d'ingérence.

Mais, en bonne doctrine, il y a là un non-sens : chacune des souverainetés postule l'autre. Ce que confirme d'ailleurs la réalité : pour faire respecter par autrui son domaine, encore faut-il en avoir le contrôle. En l'absence d'autorité supranationale capable de faire régner un minimum d'ordre dans le monde, un pays livré à l'anarchie serait une proie tentante pour ses voisins.

Par sa rigueur dogmatique, la notion de souveraineté convient donc de moins en moins à une présentation nuancée de l'autorité qu'exerce l'Etat sur son territoire et la population de celui-ci, ainsi qu'à celle de l'indépendance qu'UN Etat peut opposer aux autres. Mais, comme ce mot est encore d'un usage courant, il paraît opportun d'y avoir recours pour poser une dernière question : la France peut-elle renoncer à la souveraineté qu'elle détient sur la Corse et les DOM ? Sa constitution le lui permet-elle?

Comme toujours lorsqu'une hypothèse n'a pas été expressément prévue par les rédacteurs d'un texte juridique des solutions contradictoires sont proposées par ceux, juristes ou non, qui se livrent à l'interprétation de celui-ci. N'imaginant pas un instant qu'un département français, fût-il ultramarin, puisse un jour réclamer son indépendance, il n'est pas venu à l'esprit des constituants de 1958 de prévoir expressément cette éventualité. Qu'on se mette à leur place. Ils avaient encore à l'oreille les discours vibrants de patriotisme par lesquels Aime Césaire et les communistes réclamaient, douze ans plus tôt, la départementalisation. Et si, entre temps, ces politiciens martiniquais avaient changé leur fusil d'épaule, les revendications autonomistes du PPM devaient laisser froid un jacobin tel que Michel Debré. On s'est donc contenté de déclarer, comme d'usage, la 
République française «indivisible». Et voici que maintenant, en dehors d'Alfred Marie-Jeanne qui pense, lui, que le sort de la Martinique ne doit pas dépendre d'un texte qu'il récuse, politiciens de tous bords et juristes scrutent le texte constitutionnel, le confrontent aux précédents que seraient en ce domaine les statuts dérogatoires obtenus par la Nouvelle-Calédonie et la Corse, pour argumenter en faveur de la solution qu'eux-mêmes préconisent pour les DOM.

Le verrou à faire sauter d'abord est celui, apparemment indestructible, que représentent les principes d'unité et d'indivisibilité de la République. Or il est facile de démontrer que celle-ci n'est pas « une » : les collectivités territoriales ne manquent pas qui, en France métropolitaine même, bénéficient de statuts spéciaux, tels Paris et l'Alsace. Mais cette première escarmouche est superflue puisque l'article 2 de la constitution de 1958 ne reprend pas, avec raison, le premier adjectif de la formule rituelle ${ }^{44}$. Puis on s'attaque à celui qui y figure, « indivisible », soit pour conclure que le sens maintenant donné à ce mot est très proche de celui de «une », dont on a vu qu'il ne correspond pas à la réalité, soit pour soutenir que «l'indivisibilité de l'Etat n'est pas juridiquement opposée à la délégation normative », tout en reconnaissant plus loin que l'autonomie politique ainsi concédée est une « antichambre de l'indépendance $» 45$.

44. Elle est apparue dans la constitution de 1791 qui déclare le royaume « un et indivisible » $\left(\right.$ art. $1^{\text {er }}$ du T. II, mal à propos intitulé : «De la division du royaume...») pour mettre un terme solennel à la diversité des statuts des provinces sous l'Ancien Régime. Elle fut reprise à l'art. $1^{\text {er }}$ de la constitution de l'An I, où elle consacrait la victoire du Jacobinisme sur le Girondisme décentralisateur à l'extrême. Elle sera ensuite traditionnellement répétée dans les constitutions de l'An III (art. $1^{\text {er }}$ ), de l'An VIII (art. $1^{\text {er }}$ ) et de 1848 (Préambule, art. II). Ignorée par celle de 1875, en raison des circonstances dans lesquelles elle fut élaborée, elle sera reprise en 1946, mais sous sa forme réduite à l'indivisibilité $\left(\mathrm{T} .1^{\mathrm{er}}\right.$, art. $\left.1^{\mathrm{er}}\right)$ puis, complète, dans l'art. 85 , au T. X, Des collectivités territoriales, qui ne prévoit expressément aucune possibilité de sécession de l'une de celles-ci. Au contraire, le T. XII, Des collectivités locales de la constitution de 1958, dont l'art. 76 initial formulait clairement cette possibilité, ne reprend pas l'énoncé de ce principe. Mais la contradiction avec l'art. 2 est certaine.

45. G. Rossinyol, «Les accords de Nouméa du 5 mai 1998 : un nouveau statut pour la Nouvelle-Calédonie », in RDP, 2000, n² 2, p. 477 et 483-485. 
On fait aussi valoir dans le même but que la décentralisation administrative n'a pas de limite juridique, qu'elle peut conduire à accorder à certaines collectivités territoriales une autonomie comprise au sens étymologique du mot, c'est-à-dire «un pouvoir normatif initial qui est un pouvoir législatif et pas seulement réglementaire ». Et de citer à l'appui de cette thèse les exemples de l'Espagne et de l'Italie. L'indivisibilité de l'Etat se réduirait à exclure «l'autonomie constitutionnelle ${ }^{46}$. Mais c'est méconnaître la limite à la fois juridique et logique de la décentralisation, abrégé de l'expression «décentralisation administrative ». En dépit de l'absence de toute définition dans la constitution ou les lois, dans un régime de séparation des pouvoirs, la décentralisation a les mêmes limites que le pouvoir exécutif et ne peut donc excéder celles du pouvoir réglementaire ${ }^{47}$. Une délégation normative initiale partielle entraîne inévitablement un partage, non pas de la souverainetée ${ }^{48}$, mais du pouvoir législatif, une situation d'attente qui doit normalement conduire à l'indépendance de la collectivité autonome, à moins que, comme l'écrit Anne-Marie Le Pourhiet, «l'autonomie assistée et le

46. Op. cit., p. 477.

47. Contrairement à ce qu'écrit $\mathrm{O}$. Audeoud, «Les collectivités infra-étatiques dans la vie internationale », in L'Etat souverain... op. cit., p. 153-154, fédéralisme et décentralisation ne conduisent pas à une « distribution de compétence similaires ». Dans le fédéralisme, à défaut de la souveraineté, le pouvoir législatif est partagé. Il ne l'est pas dans la décentralisation. Et si L. Favoreu, cité par l'auteur, a raison d'écrire qu'il y a toute une gamme de situations possibles entre Etat unitaire et Etat fédéral, le critère du pouvoir législatif - partagé ou monopolisé - permet de distinguer clairement l'Etat fédéral pur, tel que les Etats-Unis, de l'Etat unitaire pur qu'était encore naguère la France. En droit, comme dans les disciplines dites exactes. il importe de suivre une démarche scientifique dont la base est le sens des mots. Ce qui n'interdit en rien de formuler les nuances que la réalité impose. Restreindre le sens du mot «Etat », dilater au maximum celui de «souveraineté » ou de « régionalisation » ne peut conduire qu'à la confusion. C'est ce que redoutait Javolenus, cité supra, en exergue.

48. L'opinion contraire exprimée par J.Y. Faberon, «La Nouvelle-Calédonie, pays à souveraineté partagée », in RDP, 1998, $\mathrm{n}^{\circ} 3$, p. 645-648 repose sur une conception erronée de la souveraineté. 
fédéralisme parasitaire » ne soient « les meilleurs garants » du recul devant le dernier pas à franchir ${ }^{49}$.

Quant aux textes constituant le siège de la matière, ceux relatifs aux DOM et aux TOM, on pouvait certes affirmer jusqu'à la loi constitutionnelle du 4 août 1995 que l'article 76 permettait la sécession d'un TOM. Mais l'abrogation de cet article réduit cette possibilité à ce que prévoit le $\S 2$ de l'article 74 (LC du 25 juin 1992, art. 3) que l'on peut, à l'extrême rigueur, interpréter dans ce sens : «Les statuts des territoires d'Outre-mer sont fixés par des lois organiques... et modifiés, dans la même forme, après consultation de l'assemblée territoriale intéressée ». Pourtant, si l'on rapproche ce texte de celui de l'article 74 qui accorde aux TOM une « organisation particulière » alors que l'article 73 n'offre aux DOM que «des mesures d'adaptation nécessitées par leur situation particulière ", on ne peut qu'en conclure que les dispositions concernant les premiers ne sauraient être étendues aux seconds. Comme l'écrit Guy Carcassonne, «Les DOM sont des départements avant d'être d'Outre-mer $»^{50}$. La constitution exclut donc toute application aux DOM du statut concédé à la Nouvelle-Calédonie, TOM, lors des accords de Matignon confirmés par la loi constitutionnelle du 20 juillet 1998 et insérés au titre XIII ${ }^{51}$.

En revanche, force est bien de reconnaître que le récent compromis de Matignon sur la Corse serait susceptible, lui, de devenir un dangereux précédent si, initiative personnelle du premier ministre contestée au sein même du gouvernement et dans l'opinion publique, y

49. A.-M. Le Pourhiet, « Nouvelle-Calédonie : la nouvelle mésaventure du positivisme », RDP, 1999, $\mathrm{n}^{\circ} 4$, p. 1019. Avec raison, l'auteur fait remarquer que, l'Etat subventionnant l'exercice des compétences transférées, la Nouvelle-Calédonie va, comme les DOM, s'habituer à vivre au-dessus de ses moyens. La société évoluera selon le modèle martiniquais : les Caldoches domineront la production et le commerce tandis que les Mélanésiens monopoliseront la fonction publique.

50. La constitution, introduite et commentée par G. Carcassonne, $3^{\mathrm{e}}$ éd., 1999, p. 314.

51. Dispositions transitoires relatives à la Nouvelle-Calédonie. Ne peut non plus être invoquée comme précédent la prochaine transformation de Mayotte de collectivité territoriale en collectivité départementale, que les Mahorais ont approuvée par référendum le 2 juillet 2000 . Il s'agit en effet d'une intégration plus poussée et non de la préparation d'une sécession. 
compris celle des Ajacciens, il n'était manifestement contraire à la Constitution en privant de toute signification l'art. 2 de celle-ci.

La solution de ce problème d'interprétation n'est donc pas juridique mais politique. Elle se présente sous la forme d'une alternative entre les deux termes de laquelle il est urgent de choisir.

Ou bien, pour éviter les désordres, voire le terrorisme, on juge souhaitable l'indépendance à court ou moyen terme de la Corse et des DOM, et on se réfère à l'interprétation laxiste de la Constitution ou, mieux, on procède une fois de plus a la révision de celle-ci. Cette solution ne ferait qu'accélérer le processus de désagrégation que va subir la France du fait de la suprématie européenne et de la décentralisation.

Ou bien l'emporte une réaction souverainiste provoquée par la perspective de cette débandade et il convient alors d'accorder à ces départements un statut de large autonomie dans la limite de la compétence administrative. Est au contraire à proscrire l'octroi d'un pouvoir législatif, même limité et sous contrôle parlementaire, car l'utilisation de ce veto provoquerait un mécontentement comparable a celui qui entraîna jadis la destitution de Louis XVI.

Les Martiniquais souhaitent-ils connaître la rigueur politique et économique cubaine ou conserver leur confort en substituant à l'assistance $^{52}$ française celle de l'Europe, qui risque d'être nettement plus directive ? Il ne semble pas pour l'instant et sans doute la classe politique locale dansera-t-elle encore longtemps la valse-hésitation qui fait clamer à tel ou tel son « ras le bol des révolutionnaires sans révolution, des poseurs de bombes sans bombe, des indépendantistes dans le cadre de la république française $»^{53}$.

52. La mode est d'employer à ce propos le mot « assistanat ». A tort, ce mot désigne exclusivement la fonction d'assistant, d'un professeur, par exemple.

53. Propos de Tony Delsham, couverture d'Antilla, n 891, 3 juin 2000. 
Quant à la notion d'Etat, on ne saurait partager l'opinion des juristes qui louent son «étonnante souplesse »" Il s'agit en réalité d'une falsification de cette idée, comme de celle de souveraineté, pour une raison politique et morale jugée supérieure. L'ONU confère à des collectivités inorganisées la qualité d'Etat qui les met théoriquement à l'abri de toute ingérence, dans l'espoir que cet avantage leur permettra de remplir plus tard les conditions jadis exigées pour être reconnu comme tel. On peut toujours l'espérer, mais le moins que l'on doive dire est qu'elles n'en prennent pas le chemin.

Il faut le reconnaître franchement et sans condescendance aucune: il existe dans le monde, en cette fin du $\mathrm{XX}^{\mathrm{e}}$ siècle, des populations qui ne sont pas faites pour s'organiser et vivre selon le schéma européen de l'Etat, et encore moins de l'Etat de droit. Accablées par la misère, la maladie, les massacres interethniques, attachées à leurs modes de vie ancestraux mais aussi tentées par les délices de la société de consommation, elles vivent au jour le jour, sans espoir d'amélioration de leur sort. A moins qu'elles ne se laissent mourir, comme les Mabris, peuple de la jungle laotienne dont le mode de vie est antérieur au néolithique. Qui trouvera le juste équilibre entre, d'une part, le souci et même le devoir d'aider ces peuples et, d'autre part, celui de leur apporter exactement la dose de progrès qui convient à leur situation, sans leur imposer le modèle occidental ${ }^{55}$.

Etonnante souplesse? Non. redoutable dureté de la notion d'Etat...

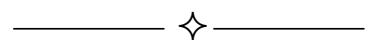

54. Propos de M. Virally, cités par J.-D. Moutons, op. cit., p. 92.

55. Pour atteindre ce but, Th. Michalon, « A la recherche de la légitimité de l'Etat», RFDC, $\mathrm{n}^{\circ} 24,1998$, p. 297-298, propose de «fonder des institutions renouvelées sur les solidarités existantes ». 


\section{Résumé}

Cet article explore la notion d'Etat, sous ses deux aspects de personne morale d'une part, d'appareil gouvernemental d'autre part, en s'interrogeant sur la capacité de la Martinique à constituer un éventuel Etat. Examinant en premier lieu les éléments constitutifs de l'Etatpersonne morale, l'auteur s'interroge sur l'existence d'une nation martiniquaise. Examinant ensuite le pouvoir détenu par l'Etat-ensemble d'institutions, à savoir la souveraineté, il dénonce son démantèlement contemporain par l'intégration de la France dans l'Union européenne et par son éclatement en cours entre une pluralité de collectivités territoriales dotées de l'autonomie.

\section{Abstract}

This paper focuses on the concept of State, regarded as 1) a legal entity, 2) a governmental apparatus, while inquiring on the ability of Martinique to eventually become a State. Looking first at the components of the legal entity, the paper wonders whether Martinique is, or not, a nation. Looking then at the main power granted to the governmental apparatus, i.e. sovereignty, it shows how it is currently being torn down, both by the building of European Union and by the eventual blowing up of French Republic into autonomous regions.
Mots-clés
Etat - Nation - Martinique - Souveraineté - Autonomie

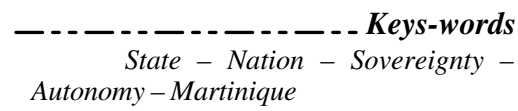

